\title{
Auf der Suche nach den "richtigen« Indikatoren
}

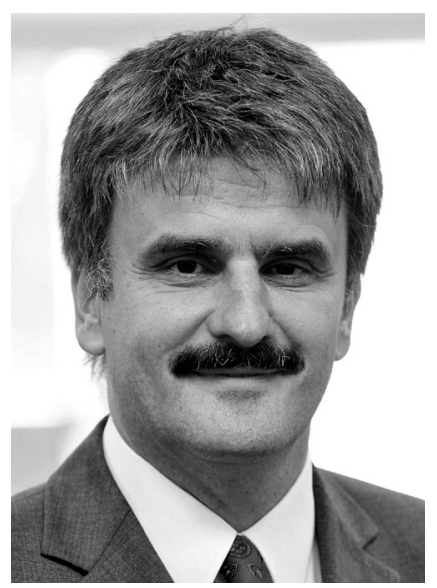

VON MICHAEL WEBER

Dr. Michael Weber ist Geschäftsführer des Heilpädagogischen Zentrums Krefeld - Kreis Viersen gGmbH, einer Komplexeinrichtung für behinderte Menschen. Zuvor war er unter anderem Leiter der Personalentwicklung beim Diözesan-Caritasverband für das Erzbistum Köln sowie Geschäftsführer des Instituts für Qualität und Wirtschaftlichkeit im Gesundheitswesen. m.weber@hpzkrefeld.de

\author{
Bei der Auswahl geeigneter Wirkungsnachweise \\ für soziale Dienstleistungen sollte es weniger um \\ die "wissenschaftlich richtigen" Indikatoren gehen, \\ sondern um Nachweise für den politischen Diskurs. \\ Diese Anschlussfähigkeit lässt sich zwar derzeit am \\ ehesten mit den Mitteln ökonomischer Evaluationen \\ herstellen, einbezogen in eine umfassende Sozialbilanz \\ sollten jedoch auch Kriterien der "Lebensqualität" \\ der Nutzer sozialer Dienste und Einrichtungen.
}

Das Thema erscheint auf den ersten Blick unproblematisch, denn Nachweise über die Wirkung der Arbeit von Dienstleistungsunternehmen für Menschen mit Behinderung existieren bereits.

So haben beispielsweise die rheinischen Werkstätten für behinderte Menschen mit dem überörtlichen Sozialhilfeträger (Landschaftsverband Rheinland) im Jahr 2011 eine Zielvereinbarung abgeschlossen. Darin werden der Anteil betriebsintegrierter Arbeitsplätze (ca. fünf Prozent aller Werkstattplätze) und die zu realisierende Übergangsquote behinderter Werkstattmitarbeiter auf den ersten Arbeitsmarkt (ca. fünf Promille aller Werkstattplätze) als Wirkungskennzahlen genutzt, um den Zielerreichungsgrad des rehabilitativen Auftrags der Werkstätten zu spezifizieren.

Was im Kontext einer aktivierenden Arbeitsmarktpolitik noch halbwegs plausibel erscheinen mag - nämlich Arbeitsmarktintegration als primäres Ziel öffentlich geförderter Beschäftigung zu konzipieren (vgl. Sommer/Rosenthal 2013) -, ist für die Zielgruppe von Werkstätten durchaus fragwürdig. Denn der weitaus überwiegende Teil von Menschen mit einer "wesentlichen « Behinderung arbeitet in Werkstätten, weil eine Beschäftigung auf dem ersten Arbeitsmarkt für sie nicht möglich ist. Kennzahlen über die Wirkung von Werkstattarbeit existieren also nur für circa sechs Prozent der Klienten
(Summe der Übergänge auf den ersten Arbeitsmarkt und der betriebsintegrierten Arbeitsplätze). Für die restlichen 94 Prozent werden auf der Steuerungsebene Kostenträger-Leistungserbringer keine Aussagen getroffen, sieht man einmal ab von Nebenthemen wie Teilzeitbeschäftigung oder Mobilität, die im Wesentlichen die pekuniären Interessen des Kostenträgers an der Reduzierung von Leistungsentgelten und Fahrtkosten bedienen.

Die offenkundige Simplizität, die in diesem Konzept von Wirkungsindikatoren zum Ausdruck kommt, hat der Soziologe Klaus Türk vor vielen Jahren als ein pathologisches Grundmuster von Organisationen ausgedeutet (vgl. Türk 1976). Werden aus der Umwelt zu viele und vor allem: zu widersprüchliche Signale empfangen, tendieren Organisationen zur Übersteuerung und greifen zu rigiden Vereinfachungen.

Für Werkstätten für behinderte Menschen heißt das: Aussagen über die Wirkung von Werkstattarbeit werden auf den Zielaspekt der Arbeitsmarktintegration verengt. In den aktuellen behinderungspolitischen Diskursen können auf diesem Wege immerhin die hochgradig diffusen Anforderungen an eine Inklusion von Menschen mit Behinderung in die Gesellschaft bedient werden.

Das Motto lautet: je höher die Übergangsquote, desto inklusiver die Einrich- 
tung. Der weitaus größte Teil aller Werkstattmitarbeiter, denen eine Beschäftigungsfähigkeit auf dem ersten Arbeitsmarkt abgeht, fällt in diesen Diskursen freilich durch das Raster der politischen Aufmerksamkeit. So eben noch wahrnehmbar sind die zwischen Indifferenz und Herablassung changierenden Aussagen des politischen Establishments, wonach »Werkstätten für Menschen mit Behinderung eine (...) wertvolle Arbeit leisten und auch künftig benötigt (werden)«(vgl. Bundesrat 2013, S. 5).

Was aber ist das "Wertvolle« an der Werkstattarbeit und warum sollte unsere Gesellschaft diese durchaus kostenträchtige Form der Dienstleistung weiter aufrechterhalten?

\section{Mit Social-Return-On-Investment- Kennzahlen Anschluss an (finanz-) politische Diskurse gewinnen}

Mit solchen Fragen befindet man sich im Spannungsfeld sozial- und finanzpolitischer Konzepte und Interessenlagen. Bei der Auswahl geeigneter Wirkungsnachweise von Werkstätten für behinderte Menschen geht es nach meinem Dafürhalten weniger um wissenschaftliche Reputation bei der Suche nach dem heiligen Gral der »richtigen « Wirkungs- zialwirtschaftliche Unternehmungen bestimmt (vgl. Schellberg 2010). Dabei kann mittels Wirkungsanalysen (1) deutlich gemacht werden, dass sich der gesellschaftliche Nutzen von Werkstätten eben nicht auf die Höhe der von öffentlichen Kostenträgern erstatteten Leistungsentgelten beschränkt, sondern ein quantifizierbarer Nutzen zum Beispiel auch für das regionalökonomische Umfeld von Werkstätten entsteht (direkte und induzierte Wirkungen für die regionale Beschäftigung, die regionale Nachfrage und die kommunalen Finanzen = SROI-Perspektive der regionalökonomischen Wirkung).

Anschlussfähig an die derzeitige politische Diskussion um ein Bundesteilhabegeld erweist sich das Ergebnis von Transferanalysen, wonach ein großer Teil der von kommunaler Seite erbrachten Finanzmittel (64\% aller Zuflüsse aus öffentlichen Mitteln) letztlich über die Werkstätten an den Bund (18\% der Rückflüsse) und vor allem an die Sozialversicherungsträger $(65 \%$ der Rückflüsse) »durchgereicht « werden. Die Hauptkennzahl des SROI, die öffentliche Zuschüsse und Rückflüsse an die öffentliche Hand ins Verhältnis setzt, beläuft sich auf ca. 50 Prozent; die Werkstätten »verbrauchen« also lediglich die Hälfte der öffentlichen Mittel.

\section{"Ein Großteil der für Werkstätten aufgebrachten kommunalen Mittel wird sofort an die Sozialversicherung und andere Nutznießer durchgereicht"}

indikatoren (vgl. Sawhill/Williamson 2001, S. 379), sondern zuvorderst um die Anschlussfähigkeit an politische, nicht zuletzt finanzpolitische Diskurse.

Diese Anschlussfähigkeit lässt sich derzeit am ehesten mit den Mitteln ökonomischer Evaluationen herstellen (vgl. Rauscher u. a. 2012), insbesondere mit sogenannten Kosten-Nutzen-Analysen, die sowohl den Kosten- und Investitionsaufwand der Gesellschaft für soziale Dienstleistungen als auch den gesamtgesellschaftlichen Nutzen (Outcome) monetär, also in Geldeinheiten, bewerten.

Das Konzept eines Social Return on Investment (SROI) stellt einen analytischen Rahmen zur Verfügung, der den Wertschöpfungsbeitrag von Werkstätten als so-
Der argumentative Nutzen von SROIKennzahlen wird insbesondere dann deutlich, wenn Aussagen über die vermeintliche Kostenüberlegenheit von Integrationsfirmen gegenüber Werkstätten in den politischen Raum geschickt werden (vgl. Röthig/Münning 2013). Dem Vorstandsvorsitzenden der Bundesarbeitsgemeinschaft überörtlicher Sozialhilfeträger, Matthias Münning, muss man empfehlen, sich mit den vorliegenden Daten vertraut zu machen. Danach fallen die gesellschaftlichen Nettokosten, die ja nicht mit den Kosten eines bestimmten (kommunalen) Trägers identisch sind, für Plätze in Integrationsunternehmen höher aus als in Werkstätten, zumindest dann, wenn ein Komplettwechsel aller
Werkstattplätze in Integrationsunternehmen beabsichtigt wäre.

Unter Kosten-Nutzen-Gesichtspunkten geht es stattdessen um einen intelligenten Institutionen-Mix zwischen Werkstattplätzen und Arbeitsplätzen in Integrationsunternehmen. Die quantitative Ausprägung dieses Mischungsverhältnisses sollte dabei in erster Linie das Ergebnis evidenzbasierter SROIAnalysen sein und sich weniger an eminenzbasierten Einschätzungen einzelner Politakteure ausrichten.

\section{Lebensqualität als Wirkungsindikator berücksichtigen}

Wenn es um aussagefähige Wirkungsnachweise von Werkstätten geht, sollten ökonomische Evaluationen auch in Form vergleichender Kosten-Nutzwert-Analysen erbracht werden. Die Nutzwerte werden in diesen Fällen, abweichend von den oben genannten SROI-Kennzahlen, als nicht-monetäre Größen konzipiert.

Die fachpolitisch leitende Fragestellung lautet: Woran will eine moderne Gesellschaft eigentlich erkennen, ob ein Unternehmen des ersten Arbeitsmarktes, ein Integrationsunternehmen, eine Werkstatt, eine Tagesförderstätte oder eben doch die Tagesgestaltung im Haus der Eltern oder einer Wohnstätte die geeignete Form der Teilhabe am Arbeitsleben für einen Menschen mit einer (wesentlichen) Behinderung ist?

Entscheidet man sich an diesem Punkt für die Messung von Lebensqualität als adressatenbezogenen Wirkungsindikator, kann etwas Licht in den von Inklusionsdiskursen vernebelten fachpolitischen Raum gebracht werden. Ein Instrument für die Messung von Lebensqualität, verstanden als subjektive Bewertung objektiver Lebensbedingen, liegt mittlerweile vor. (2)

Die Orientierung an dem Wirkungsindikator Lebensqualität hat unter anderem den Vorteil, dass sozialwirtschaftliche Analysen in Kontakt zu gesundheitsökonomischen Evaluationsstudien treten können, die mittlerweile neben den klassischen Kennzahlen der Morbidität und Mortalität auch Lebensqualitätsmaße berücksichtigen (bei wechselseitiger Verrechenbarkeit mit der Lebenszeit als quality adjusted life year - QUALY - darstellbar; vgl. Deutscher Ethikrat 2011, S. 37 ff.). 


\section{Literatur}

Bundesrat-Drucksache Nr. 282/12: Entschlie-

Bung des Bundesrates "Schaffung eines Bun-

desleistungsgesetzes« vom 22.03.2013.

Deutscher Ethikrat 2011: Nutzen und Kosten im Gesundheitswesen. Zur normativen Funktion ihrer Bewertung, Stellungnahme, Berlin.

Glatzer, Wolfgang 2012: Lebensqualität. Eine über Wachstum und Wohlstand hinausgehende gesellschaftliche Leitidee, in: Blätter der Wohlfahrtspflege 159, Heft 4, S. 123-129. Rauscher, Olivia/Schober, Christian/Millner, Reinhard 2012: Social Impact Measurement und Social Return on Investment (SROI)-Analyse. Wirkungsmessung neu?, Working Paper, Wien Röthig, Iris/Münning, Matthias 2013: „Wir könnten Gutes tun und dabei noch sparen «, in: Wohlfahrt Intern 8, Heft 5, S. 6-7.

Sawhill, John C./Williamson, David 2001: Mission impossible. Measuring Success in Non-Profit-Organizations, in: Nonprofit Management and Leadership 11, S. 371-386. Schellberg, Klaus-Ulrich 2010: Wertschöpfung. Sozialen Nutzen belegen, in: SOZIALwirtschaft 20, Heft 6, S. 19-22.

Schulze, Gerhard 2003: Die Beste aller Welten. Wohin bewegt sich die Gesellschaft im 21. Jahrhundert?, München.

Sommer, Jörg/Rosenthal, Peer 2013: Zehn Jahre »Hartz-Reformen«. Bilanz und Perspektiven für die öffentlich geförderte Beschäftigung, in: Theorie und Praxis der sozialen Arbeit 64, Heft 2, S. 84-92.

Türk, Klaus 1976: Grundlagen einer Pathologie der Organisation, Stuttgart.

Wagner, Britta 2013: Social Return on Investment: Gesellschaftliche Kosten und individuelle Wirkungen, in: SOZIALwirtschaft 23, Heft 5. Weber, Michael 2012: Auf der Suche nach geeigneten Indikatoren für die Steuerung von Werkstätten für behinderte Menschen in: Vierteljahresschrift für Sozialrecht (VSSR), Heft 4, S. 305-326.

Ein Lerneffekt aus den Erfahrungen dieser gesundheitsbezogenen Studien besteht für Werkstätten übrigens darin, dass die Ermittlung von Lebensqualitätsmaßen mittels Befragungen stets eine subjektive Eigenbewertung (des behinderten Mitarbeiters) und eine Fremdbewertung (durch den betreuenden Gruppenleiter) umfassen sollte. Damit entstehen zwar keine objektiv (»wahren «) Werte, wohl aber kollektive Einschätzungen, deren Relevanz für politische Verteilungs- und Finanzierungsentscheidungen auf der Hand liegt.

\section{Regelmäßige Sozialbilanzen erstellen}

Aus Sicht des sozialwirtschaftlichen Unternehmens sollten die genannten Kennzahlen jährlich in eine Sozialbilanz eingebracht werden, die, durchaus in Augenhöhe mit der klassischen Bilanz und der Gewinn- und Verlust-Rechnung, gegenüber der Öffentlichkeit und den relevanten Anspruchs- und Interessengruppen im Unternehmensumfeld vorgestellt werden kann. Eine solche Sozialbilanz enthält neben den monetären Kennzahlen der Transferanalysen und den ebenfalls in Geldeinheiten darstellbaren wirkungsbezogenen Opportunitätserträgen (Kostenvorteile gegenüber alternativen institutionellen Regelungen) auch Lebensqualitätspunkte.

Eine Bereitschaft politisch blockierter Kostenträger, an der Indikatorisierung und Bilanzierung von Werkstattleistungen in einem dialogischen Prozess mitzuwirken (vgl. Weber 2012, S. 322), ist derzeit nicht erkennbar. Leider verhalten sich auch die Spitzenverbände der Freien Wohlfahrtspflege indifferent bis abwartend. Die Profis unter den Verbandsfunktionären wissen um die Gefahren einer kennzahlenbasierten Transparenzoffensive ihrer Einrichtungen, die am Ende in den Konter rein sparpolitisch ausgerichteter Gegenspieler laufen könnte.

Die Initiative muss daher von den sozialwirtschaftlichen Unternehmen selbst ausgehen. Immerhin existieren bereits erste Ansätze einer wirkungsbezogenen Bilanzpolitik von Werkstätten, beispielsweise für die GWW - Gemeinnützige Werkstätten und Wohnstätten $\mathrm{GmbH}$ in Gärtringen. Das Heilpädagogische Zentrum Krefeld-Kreis Viersen gGmbH wird alsbald nachziehen.

\section{Die Gefahren von Steigerungsspielen vermeiden}

Auf die Gefahren einer konsequent auf Wirkungsnachweise setzenden Unternehmenspolitik soll abschließend hingewiesen werden. Worin diese Gefahr besteht, wird an der Aussage von Wolfgang Glatzer deutlich, der in den letzten Jahrzehnten die soziologische Lebensqualitätsforschung in Deutschland maßgeblich bestimmt hat:

"Nicht die Vorstellung einer optimalen Lebensqualität leitet hier (in sozialen Dienstleistungsorganisationen, M. W.) das Handeln an, sondern die Minimierung der Beeinträchtigung von individueller Lebensqualität sowie die Erhaltung der Lebensqualität unter schwierigen gesundheitlichen Bedingungen.« (Glatzer 2012, S. 128)

Die hauptamtlichen Mitarbeiter in den Werkstätten für Menschen mit Behinderung wissen trotz aller Inklusionsrhetorik, dass es für viele - sicher nicht für alle - ihrer Klienten keine großen Veränderungen im Hinblick auf ihre individuelle Leistungsfähigkeit geben wird und dass die fachliche Herausforderung rehabilitativen Handelns nicht zuletzt darin besteht, einen allzu schnellen Leistungsabfall zu verhindern. Eine solche Erfahrung passt weder in ein ökonomisches Denken der ständigen Leistungssteigerung, das zugegebenermaßen durch die Suche nach monetär bemessenen Wirkungsindikatoren unzulässig befeuert werden könnte, noch in ein pädagogisches Denken der stetigen Lernfortschritte.

All jene, die sich um die Sichtbarmachung der Qualität sozialer Dienstleistungen in der Behindertenhilfe bemühen, müssen sehr darauf achten, dass sie nicht Teil jenes dysfunktionalen gesellschaftlichen Steigerungsspiels werden, das uns der Soziologe Gerhard Schulze immer wieder eindrucksvoll vor Augen geführt hat (vgl. Schulze 2003). Sollten wir allerdings erreichen, dass sich die »Leistung « von Einrichtungen der Behindertenhilfe künftig nicht nur in Quoten von Übergängen auf den ersten Arbeitsmarkt oder von betriebsintegrierten Arbeitsplätzen bemisst, könnten wir schon zufrieden sein.

\section{Anmerkungen}

(1) Ich beziehe mich auf die Ergebnisse einer SROI-Studie, die das Beratungsunternehmen xit $\mathrm{GmbH}$ für acht Werkstattträger am Niederrhein und in Aachen mit ca. 10.000 behinderten Mitarbeitern für das Jahr 2010 durchgeführt hat (vgl. auch Wagner 2013).

(2) Es handelt sich dabei um ein Gemeinschaftsprojekt der Lebenshilfe Heinsberg e. V., des Heilpädagogischen Zentrums Krefeld-Kreis Viersen gGmbH, der Benediktushof gGmbH der Josefs-Gesellschaft und des Beratungsunternehmens xit $\mathrm{GmbH}$. 